\title{
PRELIMINARY STUDY ON BIODIVERSITY OF BUTTERFLIES IN BEYKOZ GROVE OF ISTANBUL, TURKEY
}

\author{
Kucukosmanoglu, A. \\ Department of Forest Entomology and Protection, Faculty of Forestry, Istanbul University, \\ 34473 Bahcekoy, Sariyer, Istanbul, Turkey \\ (e-mail: aliko@istanbul.edu.tr) \\ (Received $17^{\text {th }}$ Apr 2019; accepted $11^{\text {th }}$ Jul 2019)
}

\begin{abstract}
In this study, Lepidoptera species collected by sweep net in Beykoz grove, Istanbul province, Turkey during the years of 2017-2018 was evaluated. A total of 182 specimens were collected in Beykoz. According to identification results, 57 species belonging to 12 families were recorded. The family Noctuidae was represented by the highest number of species (19), followed by Geometridae (16) and Notodontidae (6).
\end{abstract}

Keywords: sweep net, butterflies, biodiversity, fauna, Beykoz.

\section{Introduction}

Lepidoptera, butterflies and moths, are very common insects and well known because of their very attractive colours and patterns on their wings. This order is recognized as one of the largest order of insects (Romoser and Stoffolana, 1994). Adults of the majority of extant species feed on nectar, the juice of overripe fruit or other liquids. Larvae of almost all species are phytophagous, and no parts of plants remain unexploited. Because of their phytophagus habits and high reproductive rate, many species are important pests (Gillott, 2005). The ecological-faunistic complex situation of butterflies is important for biodiversity.

There are several regional studies to determine the Lepidoptera fauna in Turkey (Akbulut et al., 2003; Akkuzu et al., 2007; Avci, 1997; Beskardes, 2002; Can, 2008; Cebeci, 2003; De Lattin, 1950, 1951; Graves, 1925, 1926; Hakyemez, 1994; Hesselbarth et al., 1995; Kansu, 1963; Kaygin et al., 2009; Kornosor, 1987; Mathew, 1881; Mol, 1977; Okyar and Aktac, 1998, 1999; Rebel, 1903).

With the new records published on butterfly observation sites of Butterfly Turkish (kelebek-turk.com) by butterfly observers, the number of butterfly species in the province of Istanbul is 125 . In order to determine the butterfly species diversity of Beykoz grove of Istanbul, a field study was carried out in 2017-2018.

\section{Materials and methods}

The study was conducted between the years of 2017 and 2018 in Beykoz grove located within Istanbul region (Fig. 1). Beykoz is a small holiday town on the Marmara Sea coasts in the city of Istanbul. Beykoz grove (located at $41^{\circ} 07^{\prime} 59^{\prime} \mathrm{N}, 29^{\circ} 05^{\prime} 54^{\prime \prime} \mathrm{E}$ ) covers in the form of deciduous forests, composed of various tree species and tall shrubs. In the forested area dominant trees are Platanus sp., Quercus spp., Alnus glutinosa, Acer campestre, Fraxinus excelsior, Carpinus betulus, Castanea sativa, Tilia tomentosa and Poulus tremula. Having rich flora and suitable climate conditions enabled Beykoz owning rich and divergent Biodiversity. 
Specimens were collected using a sweep net. Captured specimens were put into tightly closed killing jars immediately and brought to the laboratory for preparation and identification. Ethyl acetate was used as a killing agent. The collecting dates and locations concerning each specimen were recorded in the field note book. Specimens were pinned using insect pins (mostly, 3 - 4 - 5 sized) and were mounted on spreading boards. For identification, different studies (Fibiger, 1993; Forster and Wohlfahrt, 1971; Marini and Trentini, 1986; NHM, 2009; Savela, 2001; Spuler, 1910) were used. The Lepidoptera collections of Forest Entomology and Protection Department of Istanbul University, Faculty of Forestry were also used.

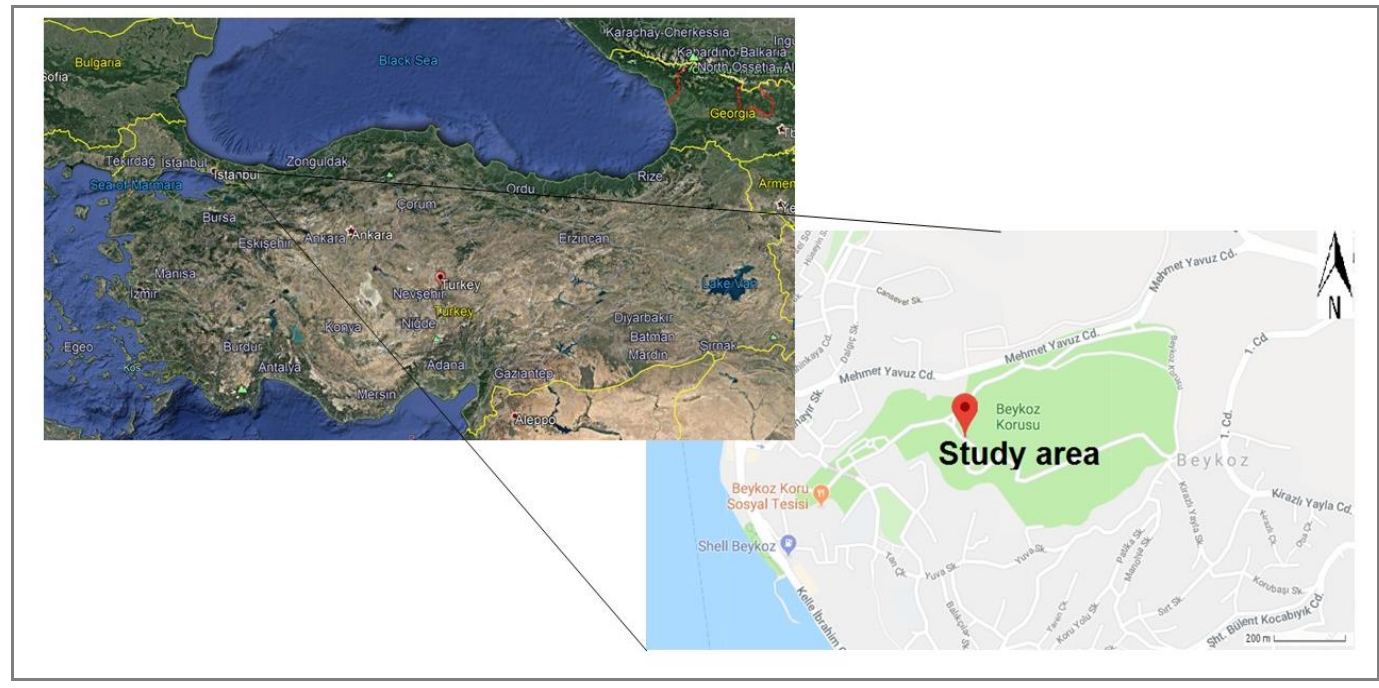

Figure 1. Location of study area

\section{Results and discussion}

A total of 182 specimens were collected from this location in Beykoz region of Istanbul. 57 species belonging to 12 families of the order Lepidoptera were identified and listed as follows. Family names and species in each family were listed as arranged alphabetically:

Order LEPIDOPTERA Linnaeus, 1758

Family COSSIDAE Leach, [1815]

Cossus cossus (Linnaeus, 1758)

Zeuzera pyrina (Linnaeus, 1761)

Family CRAMBIDAE Leach, [1815]

Cydalima perspectalis (Walker, 1859)

Family TORTRICIDAE Latreille, [1803]

Tortrix viridana (Linnaeus, 1758)

Family PYRALIDAE Latreille, 1809

Dioryctria abietella (Denis \& Schiffermüller, 1775)

Nomophila noctuella (Denis \& Schiffermüller, 1775)

Family LASIOCAMPIDAE Harris, 1841

Lasiocampa quercus (Linnaeus, 1758)

Family SATURNIIDAE Boisduval, 1837

Saturnia pavonia (Linnaeus, 1758)

Family GEOMETRIDAE Leach, [1815] 
Alcis repandata (Linnaeus, 1758)

Ascotis selenaria (Denis \& Schiffermüller, 1775)

Campaea margaritata (Linnaeus, 1767)

Cosmorhoe ocellata (Linnaeus, 1758)

Geometra papilionaria (Linnaeus, 1758)

Gnophos sartata (Treitschke, 1827)

Hemistola chrysoprasaria (Esper, 1795)

Hemithea aestivaria (Hübner, 1789)

Idaea aversata (Linnaeus, 1758)

Idaea ochrata (Scopoli, 1763)

Melanthia procellata (Denis \& Schiffermüller, 1775)

Opisthograptis luteolata (Linnaeus, 1758)

Peribatodes rhomboidaria (Denis \& Schiffermüller, 1775)

Plagodis dolabraria (Linnaeus, 1767)

Scopula nigropunctata (Hufnagel, 1767)

Selenia lunaria (Hübner, 1788)

Family NOTODONTIDAE Stephens, 1828

Drymonia dodonaea (Denis \& Schiffermüller, 1775)

Phalera bucephala (Linnaeus, 1758)

Pheosia tremula (Clerck, 1759)]

Pterostoma palpina (Clerck, 1759)

Spatalia argentina (Denis \& Schiffermüller, 1775)

Stauropus fagi (Linnaeus, 1758)

Family THAUMETOPOEIDAE Aurivillus, 1889

Thaumetopoea pityocampa (Denis \& Schiffermüller, 1775)

Family LYMANTRIIDAE Hampson, [1893]

Calliteara pudibunda (Linnaeus, 1758)

Euproctis chrysorrhoea (Linnaeus, 1758)

Lymantria dispar (Linnaeus, 1758)

Family ARCTIIDAE Leach, [1815]

Arctia villica (Linnaeus, 1758)

Miltochrista miniata (Forster, 1771)

Phragmatobia fuliginosa (Linnaeus, 1758)

Spilosoma lubricipeda (Linnaeus, 1758)

Family NOCTUIDAE (Latreille, 1809)

Agrotis cinerea (Denis \& Schiffermüller, 1775)

Agrotis ipsilon (Hufnagel, 1766)

Amphipyra pyramidea (Linnaeus, 1758)

Autographa gamma (Linnaeus, 1758)

Callopistria juventina (Stoll, 1782)

Chersotis margaritacea (Villers, 1789)

Chloantha hyperici (Denis \& Schiffermüller, 1775)

Conistra rubiginea (Denis \& Schiffermüller, 1775)

Dysgonia algira (Linnaeus, 1758)

Emmelia trabealis (Scopoli, 1763)

Epilecta linogrisea (Denis \& Schiffermüller, 1775)

Euplexia lucipara (Linnaeus, 1758)

Heliothis peltigera (Denis \& Schiffermüller, 1775) 
Herminia tarsipennalis Treitschke, 1835

Melanchra persicariae (Linnaeus, 1761)

Mythimna vitellina (Hübner, 1808)

Noctua orbona (Hufnagel, 1766)

Noctua pronuba (Linnaeus, 1758)

Xestia castanea (Esper, 1798)

A total of 182 individuals with 57 species belonging to 12 families were identified in Beykoz grove. Nymphalidae family was dominant, whereas Riodinidae had the least number of individuals. The highest number of species belongs to Noctuidae (19, $33.3 \%)$, followed by Geometridae $(16,28.1 \%)$ and Notodontidae $(6,10 \%)$. The number of species in each family (Figs. 2 and 3) and their ratios to the families were given in Table 1. In Table 2, the data concerning the capturing dates and the collecting locations about each specimen were given.

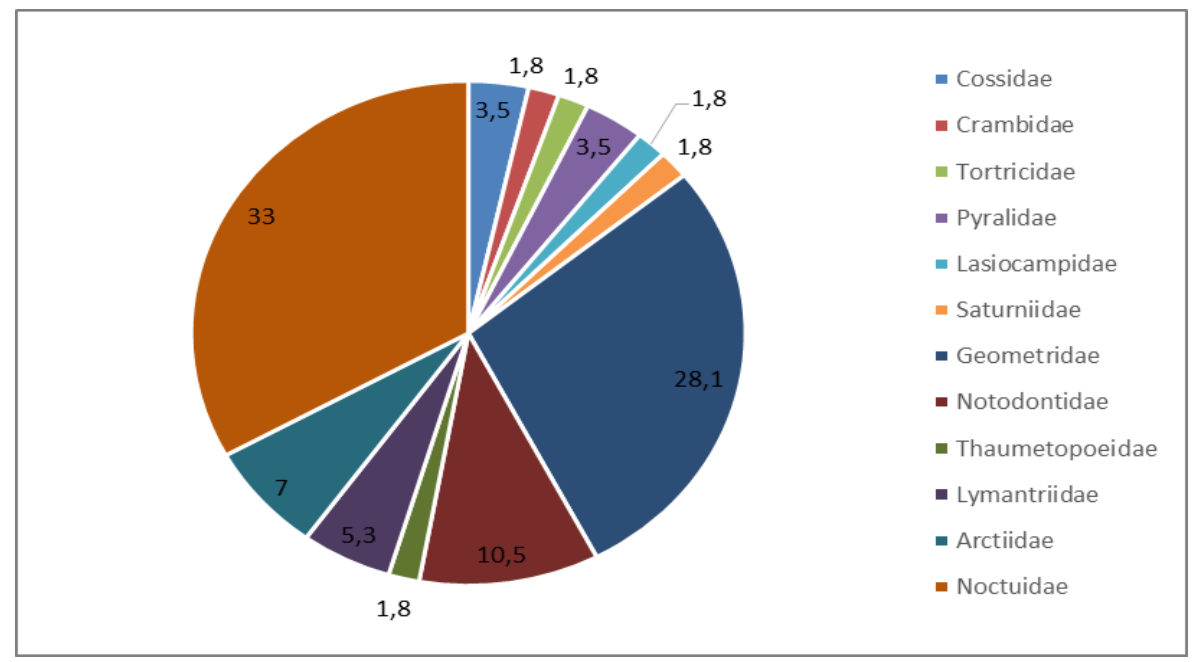

Figure 2. Percentage of species into each family

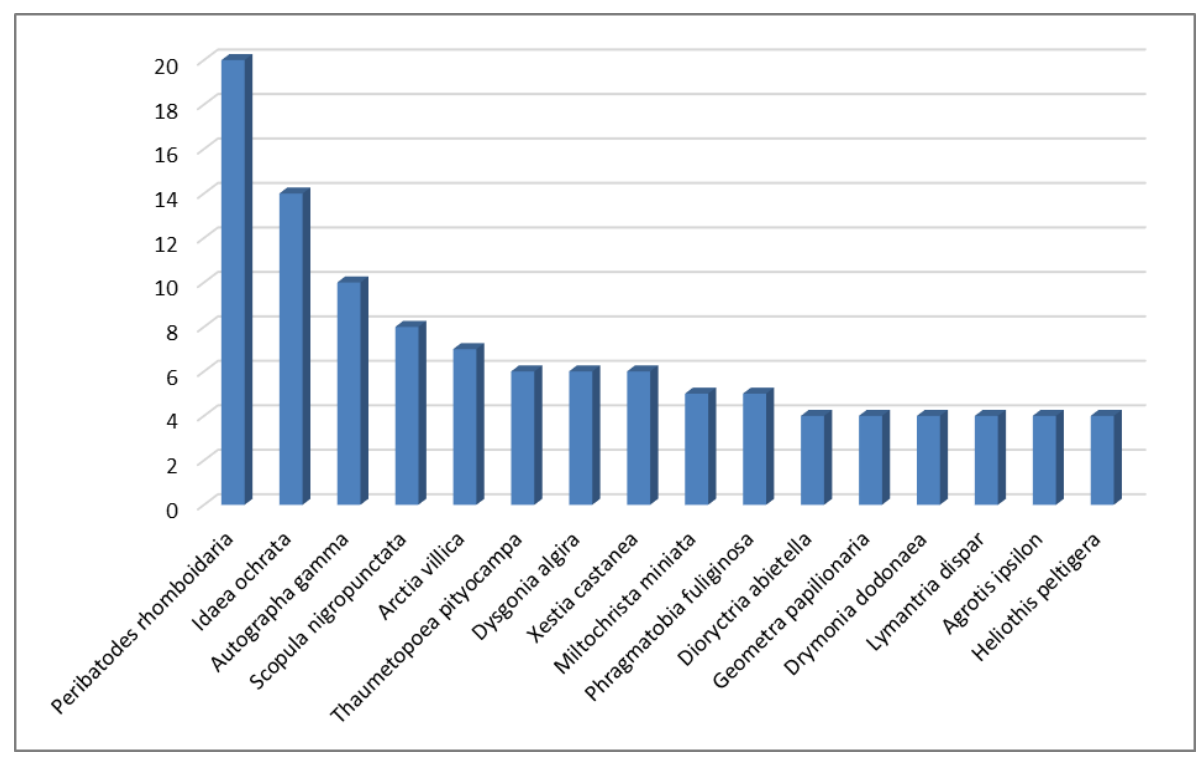

Figure 3. The total number of collected species 
Table 1. Number of species and rates of families

\begin{tabular}{c|c|c}
\hline Family & Number of species & Rate (\%) \\
\hline Cossidae & 2 & 3.5 \\
Crambidae & 1 & 1.8 \\
Tortricidae & 1 & 1.8 \\
Pyralidae & 2 & 3.5 \\
Lasiocampidae & 1 & 1.8 \\
Saturniidae & 1 & 1.8 \\
Geometridae & 16 & 28.1 \\
Notodontidae & 6 & 10.5 \\
Thaumetopoeidae & 1 & 1.8 \\
Lymantriidae & 3 & 5.3 \\
Arctiidae & 4 & 7.0 \\
Noctuidae & 19 & 33.0 \\
\hline Total & 57 & 100 \\
\hline
\end{tabular}

Table 2. The capturing dates about each specimen in Beykoz region

\begin{tabular}{|c|c|}
\hline Species & Date \\
\hline Cossus cossus & 10.06.2017 (3). Totally 3 specimens \\
\hline Zeuzera pyrina & 14.07.2017 (2); 28.06.2018 (1). Totally 3 specimens \\
\hline Tortrix viridana & 20.07.2018 (1). Totally 1 specimen \\
\hline Dioryctria abietella & 17.06.2018 (2); 19.06.2018 (2). Totally 4 specimens \\
\hline Nomophila noctuella & 20.06.2017 (1). Totally 1 specimen \\
\hline Lasiocampa quercus & 13.08.2018 (3). Totally 3 specimens \\
\hline Saturnia pavonia & 20.04.2018 (2). Totally 2 specimens \\
\hline Alcis repandata & 17.06.2017 (2). Totally 2 specimens \\
\hline Ascotis selenaria & 25.08.2018 (1). Totally 1 specimen \\
\hline Campaea margaritata & 20.07.2017 (1); 28.07.2017 (2). Totally 3 specimens \\
\hline Cosmorhoe ocellata & 18.07.2017 (1). Totally 1 specimen \\
\hline Geometra papilionaria & 15.06.2017 (4). Totally 4 specimens \\
\hline Gnophos sartata & 07.08.2018 (3). Totally 3 specimens \\
\hline Hemistola chrysoprasaria & 08.06.2018 (1). Totally 1 specimen \\
\hline Hemithea aestivaria & 28.06.2017 (1); 06.07.2018 (1). Totally 2 specimens \\
\hline Idaea aversata & 29.06.2017 (1). Totally 1 specimen \\
\hline Idaea ochrata & 29.06.2017 (2); 17.07.2017 (2); 15.07.2018 (3); 23.07.2018 (3). Totally 14 specimens \\
\hline Melanthia procellata & 22.06.2017 (1). Totally 1 specimen \\
\hline Opisthograptis luteolata & 24.05.2017 (1). Totally 1 specimen \\
\hline Peribatodes rhomboidaria & $\begin{array}{c}10.05 .2017(4) ; 16.06 .2017(5) ; 22.06 .2017(2) ; 25.07 .2018(4) ; 22.06 .2018(2) ; 23.06 .2018(3) \\
\text { Totally } 20 \text { specimens }\end{array}$ \\
\hline Plagodis dolabraria & 19.05.2017 (1); 15.06.2017 (1); 18.06.2018 (1). Totally 3 specimens \\
\hline Scopula nigropunctata & 14.06.2017 (2); 17.06.2017 (1); 22.06.2018 (1); 17.07.2018 (1); 05.08.2018 (3). Totally 8 specimens \\
\hline Selenia lunaria & 28.05.2017 (1). Totally 1 specimen \\
\hline Drymonia dodonaea & 20.05.2018 (1); 22.05.2018 (1); 24.05.2018 (2). Totally 4 specimens \\
\hline Phalera bucephala & 28.06.2017 (3). Totally 3 specimens \\
\hline Pheosia tremula & 25.09.2018 (1). Totally 1 specimen \\
\hline Pterostoma palpina & 30.08.2018 (3). Totally 3 specimens \\
\hline Spatalia argentina & 30.06.2018 (3). Totally 3 specimens \\
\hline Stauropus fagi & 20.08.2018 (2); 23.08 .2018 (1). Totally 3 specimens \\
\hline Thaumetopoea pityocampa & 18.07.2017 (6). Totally 6 specimens \\
\hline Calliteara pudibunda & 16.05.2017 (2). Totally 2 specimens \\
\hline Euproctis chrysorrhoea & 03.07.2017 (1). Totally 1 specimen \\
\hline Lymantria dispar & 03.08.2018 Avcikoru (4). Totally 4 specimens \\
\hline Arctia villica & 17.06.2017 (2); 09.05.2018 (2); 15.06.2018 (3). Totally 7 specimens \\
\hline
\end{tabular}




\begin{tabular}{|c|c|}
\hline Miltochrista miniata & 17.07.2017 (3); 18.07.2017 (2). Totally 5 specimens \\
\hline Phragmatobia fuliginosa & 20.07.2017 (3); 27.07.2017 (2). Totally 5 specimens \\
\hline Spilosoma lubricipeda & 28.06.2017 (2). Totally 2 specimens \\
\hline Agrotis cinerea & 10.08.2018 (1). Totally 1 specimen \\
\hline Agrotis ipsilon & 05.08.2018 (1); $10.08 .2018(2) ; 15.08 .2018(1))$. Totally 4 specimens \\
\hline Amphipyra pyramidea & 15.07.2018 (1) Totally 1 specimen \\
\hline Autographa gamma & $\begin{array}{c}\text { 13.06.2017 (1); } 17.06 .2017 \text { (2); } \begin{array}{c}10.08 .2018(2) ; 12.08 .2018(2) ; 14.08 .2018(3) \text {. Totally } 10 \\
\text { specimens }\end{array}\end{array}$ \\
\hline Callopistria juventina & 10.06.2017 (2). Totally 2 specimens \\
\hline Chersotis margaritacea & 23.05.2017 (1). Totally 1 specimen \\
\hline Chloantha hyperici & 22.06.2017 (1). Totally 1 specimen \\
\hline Conistra rubiginea & 10.08.2017 (1). Totally 1 specimen \\
\hline Dysgonia algira & 17.06.2017 (2); 05.07.2017 (4). Totally 6 specimens \\
\hline Emmelia trabealis & 07.07.2017 (1). Totally 1 specimens \\
\hline Epilecta linogrisea & 23.07.2017 (1). Totally 1 specimen \\
\hline Euplexia lucipara & 20.08.2018 (1). Totally 1 specimen \\
\hline Heliothis peltigera & 10.05.2017 (1); 12.06.2017 (2); 15.08.2018 (1). \\
\hline Herminia tarsipennalis & 15.06.2017 (1). Totally 1 specimen \\
\hline Lacanobia w-latinum & 02.07.2018 (1). Totally 1 specimen \\
\hline Melanchra persicariae & 20.06.2017 (1). Totally 1 specimen \\
\hline Mythimna vitellina & 20.07.2018 (1). Totally 1 specimens \\
\hline Noctua orbona & 05.08.2018 (3). Totally 3 specimens \\
\hline Noctua pronuba & 10.06.2017 (1); 22.06.2018 (2). Totally 3 specimens \\
\hline Xestia castanea & 10.07.2017 (2); 15.08.2018 (4). Totally 6 specimens \\
\hline Totally 57 species & Totally 182 specimens \\
\hline
\end{tabular}

\section{Conclusions}

In this research, 57 species belonging to 12 families of suborder Heterocera were captured and identified in Beykoz region of Istanbul. Most of the species were from three families: Noctuidae $(19,33.0 \%)$, followed by Geometridae $(16,28.1 \%)$ and Notodontidae $(6,10.5 \%)$. Among the lepidopteran species collected, 33 were considered pests for forest trees: C. cossus, Z. pyrina, T. viridana, D. abietella, $L$. quercus, S. pavonia, A. repandata, C. margaritata, G. papilionaria, $H$. aestivaria, $P$. dolabraria, S. lunaria, $H$. pyritoides, $D$. dodonaea, $P$. bucephala, $P$. tremula, $P$. palpina, S. argentina, S. fagi, T. processionea, C. pudibunda, E. chrysorrhoea, L. dispar, L. monacha, P. fuliginosa, S. lubricipeda, A. psi, A. pyramidea, B. bicolorana, C. rubiginea, L. w-latinum, M. lunaris and M. alpium (Kimber, 2009; Savela, 2001). Oak (Quercus spp.) is the dominant tree in the region and according to the literature (Kimber, 2009; Savela, 2001), C. cossus, T. viridana, L. quercus, S. pavonia, C. margaritata, $H$. aestivaria, S. lunaria, D. dodonaea, $P$. bucephala, S. argentina, $T$. processionea, C. pudibunda, E. chrysorrhoea, L. dispar, L. monacha, A. psi, A. pyramidea, B. bicolorana, C. rubiginea, L. w-latinum, M. lunaris and M. alpium caterpillars prefer oak leaves as their food plants.

$Z$. pyrina differs from these species as its larvae food regimes. The adults of $Z$. pyrina fly during June and July were collected in the end of July and in the beginning of August. L. monacha adults are different from L. dispar adults by means of morphological appearances. They often show traces of bright pink colour on the body, especially the abdomen, which however is normally concealed when at rest. L. monacha was regarded as the first record for the region. 
Most of the species obtained were collected in summer months. This was because summer is the most suitable season for the mating and regeneration activities of Lepidopteran adults.

As Kaygin et al. (2009) said: the habitats, where the butterfly and moth species have been abundantly observed should be protected and these particular locations should be preserved as butterfly protection areas. Beykoz is one of the potential butterfly protection areas.

\section{REFERENCES}

[1] Akbulut, S., Yuksel, B., Keten, A. (2003): The Lepidoptera (Insecta) Fauna of Duzce Province, Turkey. - Turk. J. Zool. 27(5): 257-268.

[2] Akkuzu, E., Ayberk, H., Inac, S. (2007): Hawk Moths (Lepidoptera: Sphingidae) of Turkey and their zoogeographical distribution. - J. Environ. Biol. 28(4): 723-730.

[3] Avci, M. (1997): Tortricidae Fauna in forests of Marmara Region. - Ph.D. Thesis, Istanbul University, Forestry Faculty, Istanbul.

[4] Beskardes, V. (2002): The butterfly and moth (Lepidoptera) species living in Istanbul Catalca Administration Forests. - M.Sc. Thesis, Istanbul University, Forestry Faculty, Istanbul.

[5] Can, F. (2008): The geometrid moths (Lepidoptera) from the Middle and Eastern Black Sea Regions of Turkey. - Turk J. Zool. 32: 351-358.

[6] Cebeci, H. H. (2003): Entomological problems in Istanbul Regional Directorate. - Ph.D. Thesis, Istanbul University, Forestry Faculty, Istanbul.

[7] Coulson, R. N., Witter, J. A. (1984): Forest Entomology (Ecology and Management). John Wiley and Sons, New York.

[8] De Lattin, G. (1950): Türkische Lepidopteren I. - Istanbul University Journal 15(4): 301 328.

[9] De Lattin, G. (1951): Türkische Lepidopteren II. - Istanbul University Journal 16(1): 4573.

[10] Fibiger, M. (1993): Noctuidae Europaeae. - Entomological Press, Soro.

[11] Forster, W., Wohlfahrt, A. (1971): Die Schmetterlinge Mitteleuropas, Eulen (Noctuidae), Band IV. - Franckhsche Verlagshandlung Stuttgart.

[12] Gillott, C. (2005): Entomology. - Springer, Dordrecht.

[13] Graves, P. P. (1925): Lepidoptera of the Constantinople. - Entomologist 63: 191-194.

[14] Graves, P. P. (1926): Heterocera from Macedonia, Gallipoli and Central Greece. Entomologist's Rec. J. Var. 38: 152-158, 165-170.

[15] Hakyemez, A. (1994): The Forest Noctuidae species of Zonguldak Regional Directorate. - Review of Faculty of Forestry, Istanbul University 44(2): 111-133.

[16] Hesselbarth, G., Van Oorschot, H., Wagener, S. (1995): Die Tagfalter der Türkei, 3 Band. - Selbstverlag Sigbert Wagener, Bocholt.

[17] Kansu, A. (1963): The list for Turkish Lepidoptera: V. - Plant Prot. Bull. 3(3): 208-223.

[18] Kaygin, A. T., Yildiz, Y., Avci, M. (2009): Lepidoptera fauna in Bartin province, in western Black Sea region of Turkey. - Afr. J. Agric. Res. 4(9): 815-822.

[19] Kimber, I. (2009): UK moths web page. - http://www.ukmoths.org.uk/ (accessed: 23 November 2009].

[20] Kornosor, S. (1987): Distribution and systematics of Noctuinae and Plusiinae (Lep. Noctuidae) species in South and Southeast Region of Turkey. - Turk. Entomol. Congress, October 13-16, İzmir, Turkey, pp. 649-659.

[21] Marini, M., Trentini, M. (1986): I Macrolepidotteri dell'appennino lucchese. - Universita degli Studi di Bologna, Instituto e Museo di Zoologia, Bologna. 
[22] Mathew, G. F. (1881): List of Lepidoptera observed in the neighbourhood of Gallipoli Turkey in 1878. - Entomologist's Mon. Mag. 18: 10-13, 29-32, 92-100.

[23] Mol, T. (1977): Gometridae species living in Marmara and Eagean Regional Forests. Rev. of the Faculty of Forestry, University of Istanbul 2329(234): 125.

[24] NHM (2009): Natural History Museum web page. http://www.nhm.ac.uk/jdsml/researchcuration/research/projects/lepindex/index.dsml (accessed: 23 November 2009).

[25] Okyar, Z., Aktac, N. (1998): Additives to Heterocera (Lepidoptera) fauna of Thracian Region. - Turk. J. Entomol. 22(1): 47-56.

[26] Okyar, Z., Aktac, N. (1999): Faunistic and Taxonomic studies on the Geometridae species of Turkish Thrace. - Turk. J. Zool. 23: 99-132.

[27] Rebel, H. (1903): Studien über die Lepidopterenfauna der Balkanlander I. (Bulgarien, Ostrumelien). - Annln. Naturh. Mus. (Wien) 18: 123-347.

[28] Romoser, S. W., Stoffolano, G. J. (1994): The Science of Entomology. - Wm. C. Brown Communications Inc., Iowa.

[29] Savela, M. (2001): Klepidoptera web page. - http://www.funet.fi/pub/ sci/bio/life/insecta/lepidoptera/index.htm (accessed: 23 November 2009).

[30] Spuler, A. (1910): Die Schmetterlinge Europas. - E. Schweizerbartsche Verlagsbuchhandlung, Stuttgart. 